\title{
Monitoramento da Qualidade da Água, Redes Sociais das Coisas e a Educação Ambiental
}

\author{
André Peres, Evandro Manara Miletto, Simone Kapusta, Telmo Ojeda \\ ${ }^{1}$ Instituto de Educação, Ciência e Tecnologia do Rio Grande do Sul (IFRS) \\ Campus Porto Alegre \\ Rua Cel Vicente, 281 - Porto Alegre, RS - Brasil \\ \{andre.peres, evandro.miletto\}@poa.ifrs.edu.br
}

\begin{abstract}
This paper presents an technological structure aiming to automatically collect, process and present environmental information online about the water quality for different user profiles. The availability of information using the raw data publishing and the principles of the Social Web Of Things allows this structure to be used for remote queries for learning, research and dissemination of socially valuable information. The characteristics of this structure are presented, experiments results in laboratory and in the real environment, as well as the considerations about the use of this kind of system in the classroom.
\end{abstract}

Resumo. Este trabalho apresenta uma estrutura tecnológica com o objetivo de automaticamente coletar, processar e apresentar informações ambientais online sobre a qualidade da água para diferentes perfis de usuário. A disponibilidade de informações usando a publicação de dados brutos e os princípios das Redes Sociais das Coisas permite que essa estrutura seja utilizada em consultas remotas para o ensino, pesquisa e difusão de informação socialmente valiosas. As características desta estrutura são apresentadas, os resultados de experiências em laboratório e no ambiente real, bem como as considerações sobre o uso deste tipo de sistema em atividades de ensino.

\section{Introdução}

A importância da conscientização e preocupação com o meio ambiente servem como fomentadores da abordagem deste tema em todos os níveis de ensino, das séries iniciais até a pós-graduação. Cabe aos professores, pesquisadores e alunos de cursos relacionados ao meio ambiente a criação do material de apoio à elaboração de diferentes ferramentas de ensino. Este material diz respeito, principalmente, aos dados brutos para análise e interpretação destes dados de forma acessível para a população tecnicamente leiga.

Considerando-se a água como um dos elementos básicos a ser analisado neste contexto, temos que, em cursos específicos de Meio Ambiente o monitoramento ambiental com foco na gestão de recursos hídricos prevê a obtenção de informações de variáveis ambientais da água tais como o pH, condutividade, oxigênio dissolvido, turbidez e temperatura, entre outras.

Geralmente o monitoramento de ambientes aquáticos é custoso em termos de infra-estrutura necessária e tempo. Estes dados são obtidos in loco com equipamentos portáteis e manipulados por profissionais, que atuam tanto na calibração, como na obtenção dos dados. 
Um exemplo da utilização de equipamentos portáteis para obtenção dos dados referentes à qualidade da água pode ser obtido em [Falleiro and Kapusta 2010]. Neste estudo, dados referentes à qualidade da água foram obtidos utilizando-se equipamentos manuais acoplados a sensores. $\mathrm{O}$ uso destes equipamentos portáteis é essencial para medições pontuais, obtendo os dados do momento da amostragem, não sendo viável um monitoramento constante (24 horas por dia, 7 dias por semana).

Para obtenção de dados ambientais a qualquer tempo é necessário que exista um equipamento autônomo capaz de realizar a coleta e envio dos dados a um sistema remoto, sem a necessidade de calibragem e acompanhamento constante.

O objetivo da presente pesquisa é o de construir uma estrutura tecnológica capaz de obter dados ambientais, armazenar, processar e publicar estes dados de forma abrangente, considerando como público alvo desde o especialista até o leigo.

O sistema WAITS (WAter quality InformaTion System), desenvolvido, provê esta estrutura através da coleta de dados realizada por dispositivos sensores, tratamento e disponibilização em diferentes interfaces. As interfaces cobrem desde a publicação dos dados brutos até a interpretação destes dados de forma coloquial. Considera-se que o sistema servirá de base para a criação de ferramentas e atividades de ensino e conscientização ambiental.

\section{Fundamentação Teórica}

Considerando o objetivo a ser atingido, o primeiro aspecto da estrutura tecnológica construída a ser abordado é a forma com a qual é possível se obter dados do ambiente.

Estudos recentes em microeletrônica tornam possível o desenvolvimento de equipamentos sensores de baixo custo, baixo consumo de energia, tamanho reduzido e capazes de conectarem-se à Internet.

Estes equipamentos podem conter sensores dos mais diversos tipos, tais como sensores térmicos, sísmicos, visuais, de radiação, umidade, movimento, luminosidade, som, entre outros [Akyildiz et al. 2002]. O objetivo destes equipamentos é o de criar um conjunto de dispositivos contendo um ou mais sensores cada (denominados nodos sensores) distribuídos no ambiente.

Analisando a bibliografia, nota-se que o monitoramento de variáveis aquáticas utilizando nodos sensores já foi realizado em diferentes pesquisas. [Ramanathan et al. 2006], por exemplo, apresenta um estudo realizado em 2006 sobre uma rede de sensores com esta finalidade: uma implantada em Bangladesh com o objetivo de identificar a presença de arsênico em água subterrânea; e outra na Califórnia para o monitoramento da propagação de nitrato também em água subterrânea. [Regan et al. 2009], relata o projeto SmartCoast do ano de 2007, o qual objetiva o monitoramento da qualidade da água em lagos, rios e estuários, além de apresentar a estrutura e os dados coletados no rio Lee na cidade de Cork (Irlanda).

Além da publicação de dados brutos, o sistema desenvolvido é utilizado em atividades de ensino. Nestas atividades, pretende-se atingir um público heterogêneo em relação à interpretação de dados ambientais. Considerando esta necessidade, o sistema WAITS publica os dados a respeito da qualidade da água em redes sociais através de um perfil específico da fonte de água. 
Em relação à utilização da informática em atividades de ensino do meio ambiente, o trabalho de [Nobre et al. 2011] destaca a importância do uso de ferramentas computacionais na conscientização ambiental desde as séries iniciais, destacando no trabalho o estudo do uso da água. O uso destas ferramentas deve considerar aspectos como a abordagem pedagógica para a faixa etária a qual se destina, conteúdos a serem explorados, grau de interatividade e facilidade de uso [Nobre et al. 2011]. No trabalho citado, foi desenvolvido um objeto de aprendizagem denominado "Consciência Ambiental"focado na água. Este objeto de aprendizagem aborda o tema através de atividades lúdicas em um ambiente virtual desenvolvido em flash, php e utilizando uma base de dados mysql.

A utilização de redes sociais para publicação de informações parte dos princípios da Internet das Coisas, como definido por [Presser et al. 2012] e das Redes Sociais das Coisas de acordo com [MacManus 2010]. A Internet das Coisas e as Redes Sociais das Coisas estão se tornando cada vez mais presentes na sociedade e seu uso em práticas de ensino é um processo natural, da mesma forma que as redes sociais já o são.

Considerando o uso de redes sociais para práticas de ensino, destacam-se os trabalhos de [Marcon et al. 2012] e [Barcelos and Batista 2012]. Em [Marcon et al. 2012] é apresentado um estudo sobre a utilização da rede social Facebook parte de uma Arquitetura Pedagógica. "O Facebook pode ser considerado uma Arquitetura Pedagógica desde que a mediação pedagógica seja intensa, guiada principalmente, mas não exclusivamente, pelo docente com objetivos definidos para ou com o grupo.'[Marcon et al. 2012]. O trabalho [Barcelos and Batista 2012] apresenta um estudo de caso analisando as dificuldades e vantagens do uso de uma Rede Social na Internet na preparação de professores.

Tanto em [Marcon et al. 2012], quanto em [Barcelos and Batista 2012], as experiências realizadas dizem respeito à utilização do Facebook como um ponto de encontro virtual para troca de informações, compartilhamento de material e discussões (como uma ferramenta de educação à distância).

\section{Metodologia de Desenvolvimento e Requisitos do Sistema}

O sistema WAITS é capaz de obter e correlacionar os diferentes dados obtidos pelos sensores, gerando informação sobre a qualidade da água monitorada. O monitoramento de dados é o ponto de partida para a criação de diferentes mecanismos de publicação. É através desta publicação que se pode despertar a curiosidade em relação as questões ambientais e consequentemente trabalhar a pesquisa e educação ambiental.

Para o compartilhamento dos dados obtidos pela rede de sensores, de acordo com o usuário final (público-alvo), foram definidas duas formas de publicação:

- publicação dos dados brutos obtidos pelos sensores de forma com que outras pesquisas envolvendo o mesmo ambiente possam aproveitá-los, que professores especialistas possam analisar diretamente para atividades de ensino, ou que sirvam de base para diferentes ferramentas e atividades de ensino;

- publicação do estado atual do ambiente através de um perfil específico em rede social (permitindo uma análise mais subjetiva da situação do ambiente) e uso em atividades de ensino menos técnicas.

O presente trabalho, de certa forma, permeia os trabalhos citados através do fornecimento de dados ambientais online (histórico e dados atuais) permitindo que trabalhos 
como [Nobre et al. 2011] possam apresentar aos alunos as condições atuais de um fluxo de água, transformando o objeto de aprendizagem em uma experiência viva e diretamente conectada com a realidade.

Da mesma forma, utiliza as redes sociais como um meio de difundir informações a serem utilizadas em práticas de ensino como em [Marcon et al. 2012] e [Barcelos and Batista 2012].

Para que se possa atingir os objetivos propostos, foi realizado um estudo para construção de um nodo sensor capaz de obter dados referentes à qualidade da água e enviar estes dados remotamente através da Internet. Os requisitos para a construção deste equipamento são: precisão dos sensores equivalente a dos equipamentos portáteis utilizados em pesquisas científicas da área de meio ambiente; possibilidade de envio de dados para Internet; alimentação via energia solar para implantação em campo; baixo custo; possibilidade de otimização/modificação do equipamento.

Após o recebimento dos dados, o sistema WAITS deve ser capaz de publicar os dados brutos de forma que possam ser utilizados por pesquisadores, professores e demais interessados. Esta divulgação deve ser feita descrevendo de forma clara: como os dados são obtidos e qual a precisão dos sensores; descrição de cada variável obtida; descrição da estrutura de dados e de como utilizá-los; exemplos de interpretação e utilização dos dados.

Para que se possa ampliar a divulgação dos dados para pessoas que não possuem conhecimento técnico específico, definiu-se que seriam criados perfis nas redes sociais Twitter e Facebook. Estes perfis são utilizados para que o sistema divulgue periodicamente a situação atual da qualidade da água monitorada. Os dois perfis são sincronizados de forma que quando o sistema realiza uma postagem no twitter, a mesma postagem é refletida no facebook.

\section{O Sistema WAITS}

O sistema WAITS tem por objetivo a criação de uma estrutura tecnológica capaz de monitorar a qualidade da água e publicar os dados e informações obtidos na internet.

A figura 1 apresenta uma visão geral do sistema na qual é possível visualizar os fluxos de dados da coleta à publicação.

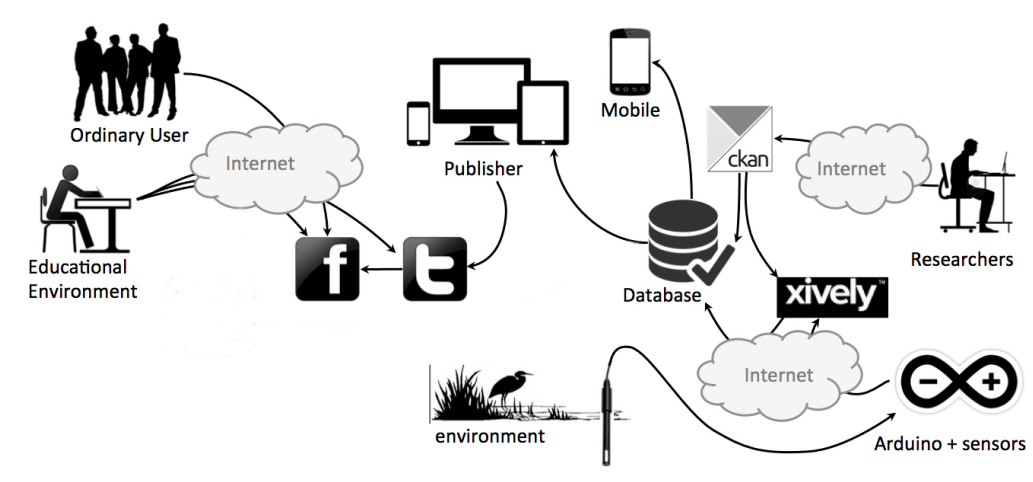

Figura 1. Visão Geral do Sistema WAITS 
Periodicamente os dados ambientais são coletados pelo nodo sensor e enviados através da Internet. A comunicação entre o nodo sensor e o sistema é realizada através de rádio-frequência (utiliza-se a rede celular GSM/GPRS) de forma que os sensores possam ser distribuídos no ambiente.

De acordo com os requisitos apontados anteriormente, optou-se pela utilização da placa controladora Arduino Mega 2560 [Arduino 2013] para construção do nodo sensor. O Arduino foi escolhido por ser uma solução de hardware aberto passível de otimização, pela flexibilidade, pela grande quantidade de módulos de hardware que podem ser acoplados, pela grande quantidade de material de apoio online e pelo baixo tempo necessário para aprendizagem e início de prototipação.

Foram utilizados sensores da empresa Atlas Scientific [Scientific 2013] para obtenção dos dados das variáveis da água acoplados ao Arduino. Para comunicação, utilizou-se um módulo GSM/GPRS. A alimentação do nodo sensor é feita através da utilização de uma placa de células de energia solar conectadas a uma bateria. Para que o nodo possa ficar na água, utilizou-se uma caixa hermética, perfurada de forma que os sensores permaneçam em contato com a água. A figura 2 apresenta o nodo sensor aberto.

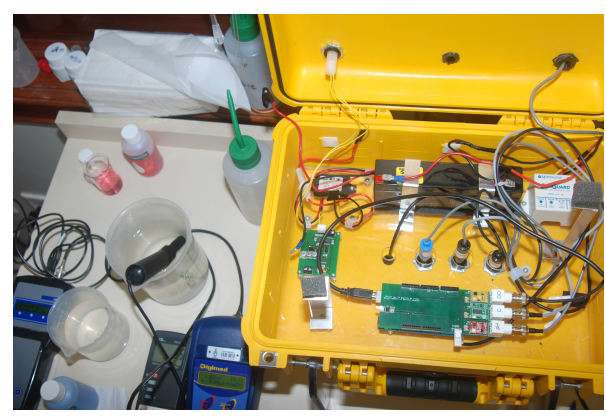

Figura 2. Nodo Sensor Aberto

Ao receber os dados do nodo sensor, os mesmos são estruturados e enviados ao sistema web Xively [Xively 2013]. O sistema Xively é utilizado como uma forma inicial de publicação dos dados obtidos além de servir como intermediário aos demais módulos do sistema. Na figura 3 é apresentada a estrutura dos dados e a interface do sistema Xively.
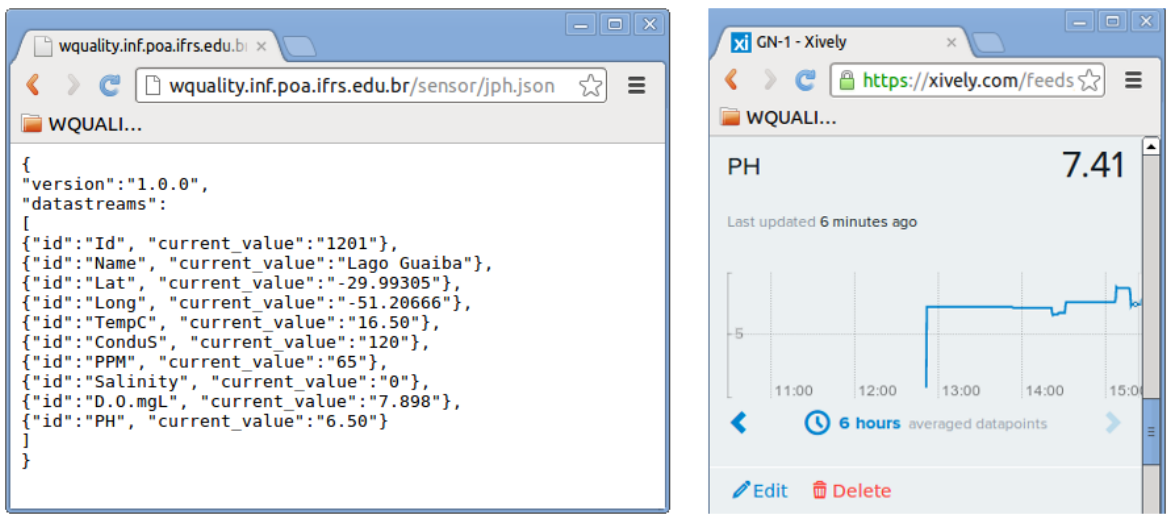

Figura 3. Estrutura JSON e Sistema Xively

É importante que os dados possuam livre acesso para uso, reuso e distribuição. 
Estas características são aplicadas no conceito de Dados Abertos. De acordo com a Open Knowledge Foundation quando se deseja utilizar dados abertos, a única exigência é a de qual a fonte dos dados seja referenciada e que qualquer forma de redistribuição dos dados siga as mesmas regras [Dietrich et al. 2012].

Ao se utilizar dados abertos, para que os dados possam ser aproveitados, é necessário que seja disponibilizada também uma descrição da forma de obtenção dos dados, como interpretá-los, qual a estrutura de armazenamento e todos os detalhes necessários para sua utilização. Com este propósito, a Open Knowledge Foundation desenvolveu o portal web CKAN [Ckan 2013]. Este portal pode ser obtido e instalado em um servidor web e funciona como uma camada intermediária entre os dados brutos e usuários.

Ao acessar o portal, é disponibilizado este detalhamento sobre os dados, e os próprios dados armazenados no Xively (o CKAN não armazena os dados). A figura 4 apresenta os dados brutos disponibilizados pelo Xively e a visualização dos mesmos dados no portal CKAN.
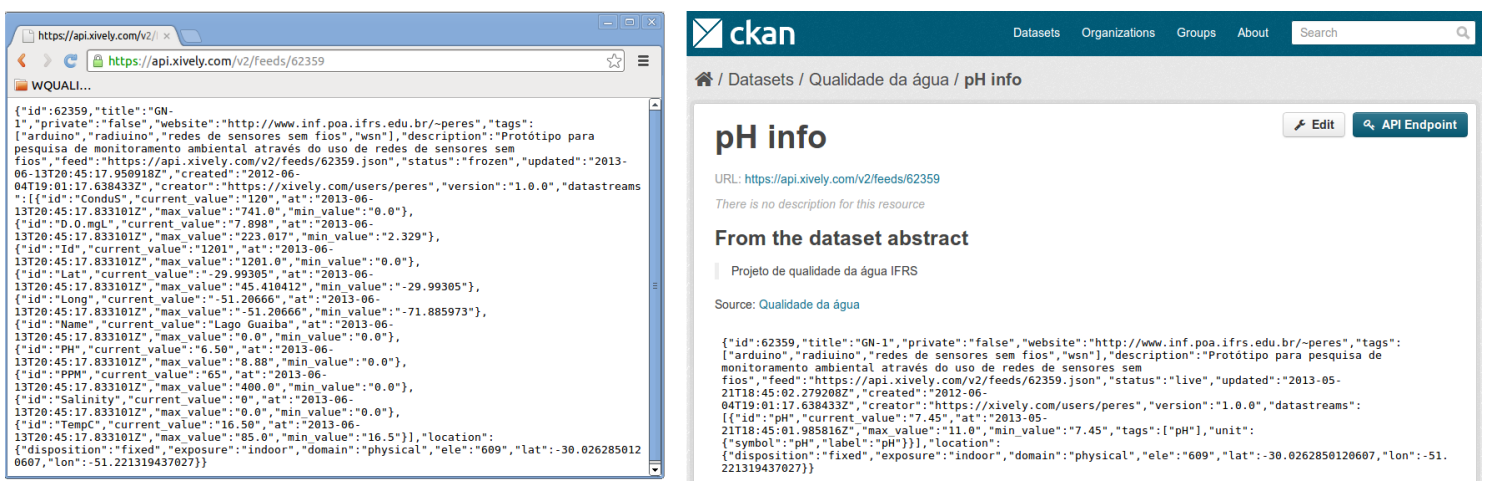

Figura 4. Dados Brutos no Xively e Portal CKAN

Durante a implantação de um novo nodo sensor, é importante que se monitore se o mesmo está operando corretamente. Se deve verificar: se os dados obtidos estão corretos; se o nodo está conseguindo enviar os dados para o sistema e; em caso de necessidade de manutenção qual a posição de um nodo. Para facilitar a implantação, foi desenvolvida uma aplicação para dispositivos móveis (neste momento está disponível para dispositivos Android) capaz de monitorar os dados obtidos pelos nodos. Este aplicativo conecta-se com o sistema WAITS para obtenção dos dados. As interfaces do sistema são apresentadas na figura 5. Apesar de ter sido desenvolvida para o momento da implantação de um nodo sensor, esta aplicação pode ser utilizada também como uma forma de monitoramento da qualidade da água. A aplicação apresenta: a posição do sensor em um mapa; a distância entre o dispositivo móvel e o sensor em metros; os últimos 5 valores obtidos pelo sensor e; uma linha de tempo com estes valores.

Para a publicação de informações nas redes sociais, inicialmente foi criado um perfil na rede social Twitter. Um módulo do sistema WAITS foi desenvolvido para obter os dados do sistema Xively, armazená-los em uma base de dados, realizar o tratamento e publicação das informações neste perfil. Após esta etapa foi criado um perfil na rede social Facebook, o qual foi sincronizado com o perfil do Twitter de forma que, ao postar a mensagem no Twitter, automaticamente a mesma mensagem é postada no Facebook. Na figura 6 pode ser visualizados os dois perfis com as informações da qualidade da água. 

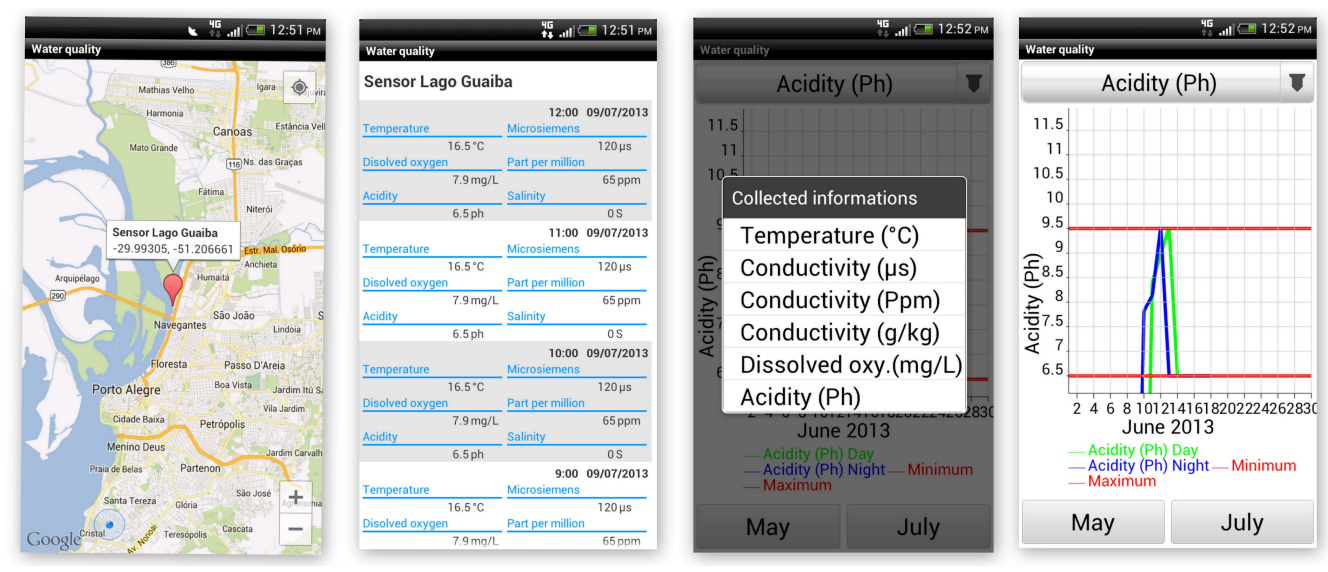

Figura 5. Aplicação Móvel
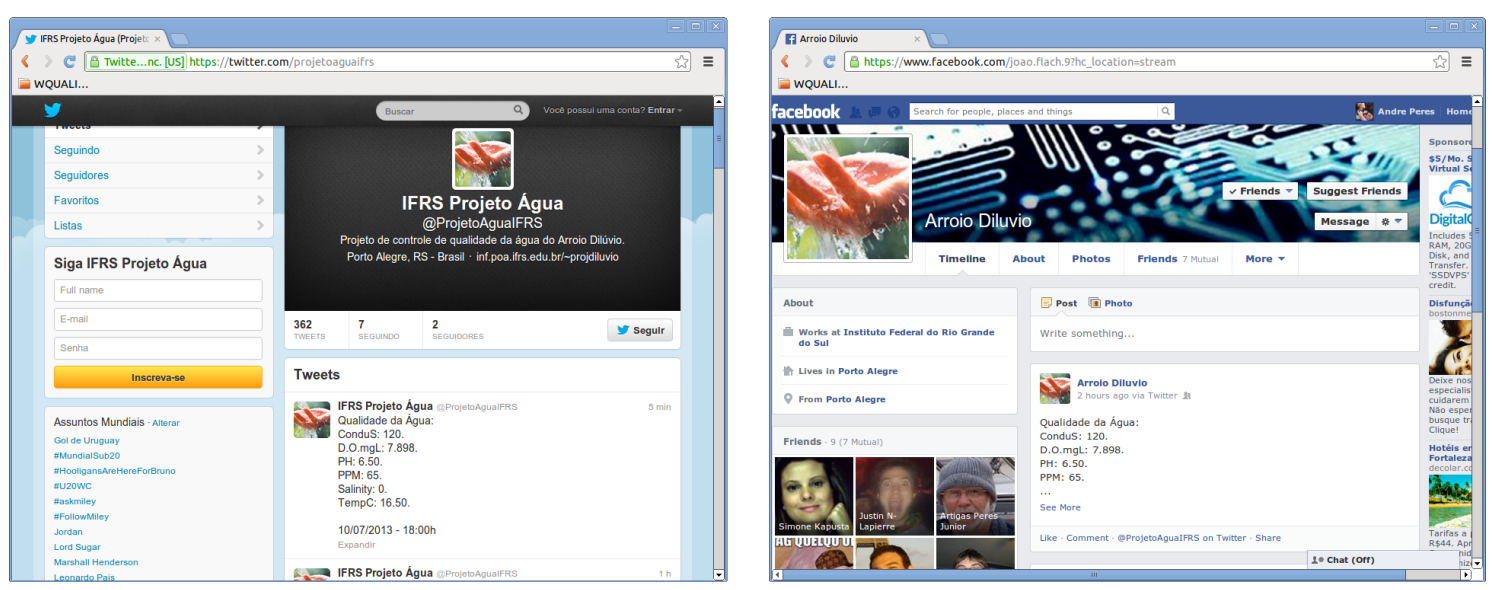

Figura 6. Perfil no Twitter e Facebook

\section{Validação do Sistema e Resultados Obtidos}

Para a validação dos resultados obtidos com o nodo sensor, foram efetuados testes em laboratório e em campo em conjunto com a utilização de equipamentos portáteis. Para tanto foram obtidos os valores das seguintes variáveis da água: condutividade $(\mu S / \mathrm{cm})$, com o condutivímetro DM 3P Digimed (Digicrom Analytical); oxigênio dissolvido $(\mathrm{mg} / \mathrm{L})$ e temperatura $\left({ }^{\circ} \mathrm{C}\right.$ ), com o uso do Oxímetro modelo DO 5519 (Lutron Eletronic Enterprise Co Ltd.) e pH com pHmetro portátil modelo MPA 210P (MSTecnopon Instrumentação). Os equipamentos foram devidamente calibrados de acordo com as instruções disponibilizadas pelos fabricantes. Os resultados do nodo sensor foram comparados com os resultados obtidos com os equipamentos portáteis.

Em laboratório, foi utilizada como amostra, $250 \mathrm{~mL}$ de água oriunda do sistema de abastecimento pública, em copo Becker. Foi utilizada uma amostra de água para os equipamentos portáteis e outra amostra para os sensores do nodo sensor. Os valores de $\mathrm{pH}$, condutividade, oxigênio dissolvido e temperatura foram obtidos com intervalos aproximados de 15 minutos, tendo sido efetuadas sete medições. Os resultados foram registrados em planilhas para posterior análise.

O experimento em campo foi efetuado no Rio Guaíba, em Porto Alegre/RS, tendo 
como ponto de apoio um píer, localizado em uma marina. Para a obtenção dos valores das variáveis da água, na camada superficial, com os equipamentos portáteis, foi utilizado um intervalo aproximado de dez minutos, entre uma medição e outra, tendo sido efetuadas nove medições. Os equipamentos portáteis eram ligados, calibrados e os sensores submergidos na água, sendo os resultados das variáveis registrados em planilhas. O nodo sensor permaneceu durante todo o período experimental na água, sendo os dados enviados para o computador. Na figura 7 pode-se visualizar os testes sendo realizados em laboratório (esquerda) e em campo (direita).
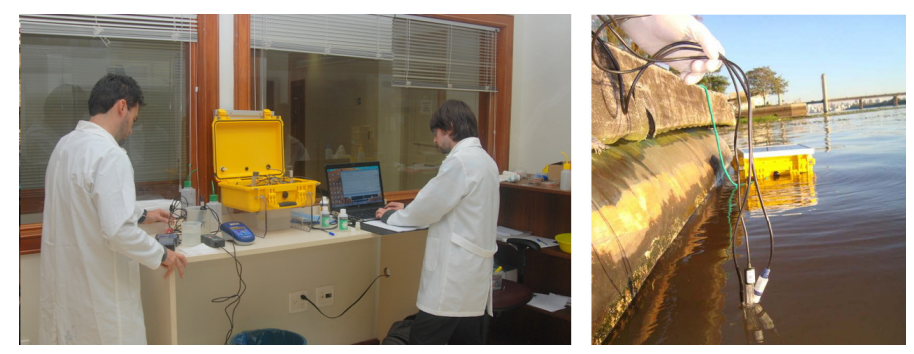

Figura 7. Experimentos em laboratório e campo

Os dados coletados foram analisados utilizando-se as ferramentas estatísticas presentes no software Bioestat versão 5.0. Inicialmente foi verificada a normalidade das variáveis com o teste de Kolmogorov Smirnov, como algumas variáveis não apresentaram distribuição normal, foi aplicado o teste não-paramétrico de Kruskal-Wallis. Para os testes foi estabelecido o nível de significância de $5 \%$, ou seja, os resultados foram considerados significativos quando $p<0,05$. Os valores de oxigênio dissolvido foram similares aos registrados pelo nodo sensor (NS) e os equipamentos portáteis (EP) no laboratório (tabela 1), não apresentando diferença significativa $(p>0,05)$.

Tabela 1. Resultados no Laboratório

\begin{tabular}{c||c|c||c|c||c|c||c|c}
\multicolumn{1}{c|}{ Tabela 1. Resultados no Laboratório } \\
\hline $\begin{array}{c}\text { Amostra } \\
\#\end{array}$ & \multicolumn{2}{c||}{$\mathbf{p H}$} & \multicolumn{2}{c|}{$\begin{array}{c}\text { Condutividade } \\
(\mu \mathrm{s} / \mathrm{cm})\end{array}$} & \multicolumn{2}{c|}{$\begin{array}{c}\text { O2 } \\
(\mathrm{mg} / \mathrm{l})\end{array}$} & \multicolumn{2}{c}{$\begin{array}{c}\text { Temp } \\
\left({ }^{\circ} \mathrm{C}\right)\end{array}$} \\
\hline & NS & EP & NS & EP & NS & EP & NS & EP \\
\hline 1 & 6,4 & 6,4 & 127 & 122,8 & 7,8 & 7,9 & 19 & 19,5 \\
\hline 2 & 6,4 & 6,5 & 127 & 122,7 & 7,5 & 7,7 & 19 & 19,5 \\
\hline 3 & 6,4 & 6,4 & 128 & 123,5 & 7,8 & 7,9 & 19,5 & 19,5 \\
\hline 4 & 6,4 & 6,4 & 128 & 123,6 & 7,8 & 7,8 & 19,5 & 19,5 \\
\hline 5 & 6,4 & 6,4 & 128 & 123,6 & 7,7 & 7,8 & 19,5 & 19,6 \\
\hline 6 & 6,5 & 6,5 & 128 & 123,6 & 7,7 & 7,8 & 19,5 & 19,6 \\
\hline 7 & 6,5 & 6,5 & 128 & 124 & 7,9 & 8 & 19,5 & 19,6 \\
\hline
\end{tabular}

Os valores de $\mathrm{pH}$ foram similares aos registrados pelo nodo sensor e pelo equipamento portátil. Em relação à condutividade, os valores registrados pelo nodo sensor foram significativamente mais elevados do que os registrados pelo equipamento portátil $(p<0,05)$. Os valores da temperatura foram significativamente menores do que os registrados pelo equipamento portátil. O resultado da temperatura provavelmente está associado com a sensibilidade da leitura do sensor do nodo.

Os valores obtidos em campo, podem ser observados na tabela 2.

Através da análise estatística, verificou-se que os valores de $\mathrm{pH}$ e de temperatura, foram similares aos registrados pelo nodo sensor e os equipamentos portáteis, não 
Tabela 2. Resultados do Experimento em Campo

\begin{tabular}{c||c|c||c|c||c|c||c|c}
\hline \multicolumn{1}{c||}{$\begin{array}{c}\text { Amostra } \\
\#\end{array}$} & \multicolumn{2}{c||}{ pH } & \multicolumn{2}{c||c}{$\begin{array}{c}\text { Condutividade } \\
(\mu S / \mathrm{cm})\end{array}$} & \multicolumn{2}{c||}{$\begin{array}{c}\text { O2 } \\
(\mathrm{mg} / \mathrm{l})\end{array}$} & \multicolumn{2}{c}{$\begin{array}{c}\text { Temp } \\
\left({ }^{\circ} \mathrm{C}\right)\end{array}$} \\
\hline & NS & EP & NS & EP & NS & EP & NS & EP \\
\hline 1 & 7,5 & 7,1 & 120 & 99,5 & 7,8 & 6,4 & 17,5 & 17,5 \\
\hline 2 & 7,3 & 7,1 & 120 & 95 & 7,8 & 7,6 & 17 & 17,2 \\
\hline 3 & 7,1 & 7,1 & 121 & 95,4 & 7,9 & 6,3 & 17 & 17,1 \\
\hline 4 & 6,9 & 7,1 & 118 & 95,7 & 7,7 & 6,2 & 17 & 17 \\
\hline 5 & 7 & 7,1 & 118 & 94,7 & 7,4 & 6,2 & 17 & 17,1 \\
\hline 6 & 6,7 & 7,1 & 120 & 92,9 & 7,8 & 6,4 & 17 & 17,1 \\
\hline 7 & 6,6 & 7,1 & 122 & 93,7 & 7,4 & 6,5 & 17 & 17 \\
\hline 8 & 6,8 & 7,1 & 120 & 93,3 & 7,8 & 6,5 & 16,5 & 17 \\
\hline 9 & 6,5 & 7,1 & 120 & 93,2 & 7,9 & 6,8 & 16,5 & 16,9 \\
\hline
\end{tabular}

apresentando diferença significativa $(p>0,05)$ entre os equipamentos utilizados. Em relação à condutividade e oxigênio, os valores registrados pelo nodo sensor foram significativamente mais elevados do que os registrados pelo equipamento portátil $(p<0,05)$. Provavelmente esse resultado do oxigênio possa ter sido influenciado pela posição mais superficial do nodo sensor, quando comparado com o do equipamento portátil.

As informações obtidas de maneira contínua poderão auxiliar os experimentos realizados pelos estudantes da área ambiental tanto para testar novas tecnologias para o tratamento de água e efluentes, bem como simular alterações ambientais em ambientes aquáticos. O tratamento das informações de forma a possibilitar a divulgação da qualidade da água de forma coloquial permite o uso deste sistema nas mais diversas atividades de ensino.

\section{Conclusões}

Esta pesquisa foca no desenvolvimento de uma estrutura tecnológica para monitoramento e divulgação de dados ambientais online de forma a serem utilizados em pesquisa científicas, ferramentas e atividades de ensino. O desafio superado foi o da integração dos diferentes níveis tecnológicos, do equipamento sensor à rede social, adequando os dados para que se possa atingir a maior abrangência de público possível.

Além de professores pesquisadores da área de computação, este projeto conta com a participação de professores pesquisadores da área de meio ambiente os quais, a partir dos resultados preliminares, indicaram uma série de vantagens na utilização do sistema WAITS em comparação com o procedimento manual. Destacam-se a possibilidade de acompanhamento online capaz de enriquecer as atividades didáticas na sala de aula através da análise e interpretação dos dados; também o acompanhamento pelos alunos via redes sociais, extrapolando os limites da sala de aula.

Como trabalhos futuros, destacam-se a necessidade de complementar a tradução dos dados obtidos para publicação nas redes sociais permitindo que um maior número de usuários possa assimilar as informações do sistema. Também é necessário ampliar os testes em campo, identificando o tempo necessário entre calibragens dos sensores (de acordo com o fabricante esta frequência é de uma vez por ano). Com a estrutura completa, pretende-se também ampliar os tipos de sensores e fornecer informações sobre outras variáveis ambientais tais como qualidade do ar, solo, etc.

Como parte das Redes Sociais das Coisas, o sistema WAITS tem a pretensão de aproximar os dados ambientais das pessoas através da rede de contatos existente nas redes 
sociais e contribuir para o enriquecimento da consciência ambiental e das atividades de ensino desta e de outras áreas.

\section{Referências}

Akyildiz, I. F., Su, W., Sankarasubramaniam, Y., and Cayirci, E. (2002). Wireless sensor networks: a survey. Computer networks, 38(4):393-422.

Arduino (2013). Arduino home page. Disponível por www em: http://www.arduino.cc.

Barcelos, G. T. and Batista, S. C. F. (2012). Rede social na internet como apoio a formação docente. In Anais do Simpósio Brasileiro de Informática na Educação, volume 23.

Ckan (2013). Ckan the open source data portal software. Disponível por www em: http://ckan.org/.

Dietrich, D., Gray, J., McNamara, T., Poikola, A., Pollock, R., Tait, J., and Zijlstra, T. (2012). Open data handbook. Disponível por www em: http://opendatahandbook.org/.

Falleiro, A. M. and Kapusta, S. C. (2010). Caracterização da qualidade da água dos arroios dilúvio e salso no município de porto alegre, rs, na estação de primavera. In Anais da 11 Mostra de Trabalhos de Iniciação Técnico-Científica, Porto Alegre, Brazil., volume 1.

MacManus, R. (2010). Social network for things. Disponível por www em: http://readwrite.com/2010/02/01/social_networks_for_things.

Marcon, K., Machado, J. B., and Carvalho, M. J. S. (2012). Arquiteturas pedagógicas e redes sociais: Uma experiência no facebook. In Anais do Simpósio Brasileiro de Informática na Educação, volume 23.

Nobre, I. A. M., do Rosário, K., Siqueira, A. L., and do Nascimento, E. L. (2011). Consciência ambiental: Objeto de aprendizagem como apoio ao ensino de meio ambiente. In Anais do Simpósio Brasileiro de Informática na Educação, volume 1.

Presser, M., Krco, S., Kowatsch, T., Maass, W., Lange, S., Carrez, F., Hunt, B., Egan, R., Höller, J., Bassi, A., et al. (2012). Inspiring the internet of things: The internet of things comic book (special edition)[ebook].

Ramanathan, N., Harmon, T., Balzano, L., Estrin, D., Hansen, M., Jay, J., Kaiser, W., and Sukhatme, G. (2006). Designing wireless sensor networks as a shared resource for sustainable development. In in Information and Communication Technologies and Development.

Regan, F., Lawler, A., and McCarthy, A. (2009). SmartCoast Project- Smart Water Quality Monitoring System. Environmental Protection Agency, Ireland.

Scientific, A. (2013). Atlas scientific home page. Disponível por www em: http://atlasscientific.com/.

Xively (2013). Xively system. Disponível por www em: https://xively.com/. 